\section{Clinical management of spasticity}

\section{A J Thompson, L Jarrett, L Lockley, J Marsden, V L Stevenson}

\section{Physical and pharmacological treatments can reduce pain and discomfort without compromising function}

S pasticity is a common symptom seen in many neurological conditions, notably head injury, spinal cord injury, stroke, cerebral palsy, and multiple sclerosis. It is also the dominant feature in several rarer conditions such as tropical and hereditary spastic paraparesis. The fact that it is relevant to many chronic neurological conditions and that the absence of multidisciplinary input can result in progressive disability makes it an ideal model to reflect service provision In the future more long term care for such patients will be done in primary care and the community. It is therefore essential that a multidisciplinary approach is used with successful liaison between secondary, primary, and social care.

Optimum management of spasticity is dependent on an understanding of its underlying physiology, an awareness of its natural history, an appreciation of the impact on the patient, and a comprehensive approach to minimising that impact which is both multidisciplinary and consistent over time. Regrettably, these essential requirements are rarely met; consequently, inadequately managed spasticity results in a range of painful and disabling sequelae which, with the right approach, are for the most part preventable.

\section{PATHOPHYSIOLOGY OF SPASTICITY AND SPASMS}

Stretch reflexes in healthy subjects are complex. At rest the reflex response is mediated through IA afferents that connect monosynaptically with the parent motor neurone. However, with the muscle activated other reflex components may also be elicited through group II spindle afferents and transcortical pathways. Stretch reflexes are normally modulated by task and during the different phases of walking. Such modulation reflects changes in motoneuronal and spinal cord inhibitory interneuronal activity. This inhibitory influence is in turn controlled by descending and peripheral inputs. In spasticity an enhanced and prolonged response to muscle stretch is seen at rest. Here both group IA and group II afferents may play a role in eliciting the response, which may be processed by monosynaptic and polysynaptic circuits. Additionally, decreased task and phase dependent modulation of stretch reflexes occurs, reflecting abnormalities of supraspinal control.

Although spasticity is seen after an upper motor neurone (UMN) lesion, the relative importance of individual descending pathways remains unclear. ${ }^{1} \mathrm{~A}$ lack of descending control over spinal cord interneuronal circuits results in a decrease in the effectiveness of spinal inhibitory circuits such as those mediating reciprocal, presynaptic, and recurrent inhibition. However, the slow clinical development of spasticity following a neurological insult argues against it simply being a release phenomenon. ${ }^{23}$ Recent studies suggest that intrinsic changes in the motor neurone develop over time following a lesion. These result in abnormally long plateaulike potentials that prolong motor neurone discharge and thus muscle contraction in response to synaptic inputs. $^{4-8}$ Although prolonged motor neurone discharge can occur in healthy subjects, the paucity of inhibitory spinal cord control in people with spasticity means that this activity could, once triggered, continue relatively unabated.

As well as hyperexcitable stretch reflexes, connective tissue changes and abnormal co-contraction can contribute significantly to stiffness. ${ }^{9}$ Connective tissue changes can occur without any contracture and the resulting stiffness may vary in a velocity dependent manner similar to spasticity, making it difficult to distinguish clinically between hypertonia of neural and nonneural origin. This distinction is, however, clinically important as the treatment of such stiffness is through physical adjuncts such as stretching and splinting rather than through pharmacological interventions.

Abnormal co-contraction can be seen after stroke during tasks that do not require any joint movement and thus stretch related activity. ${ }^{10}$ Co-contraction and excessive muscle activity during movements may therefore reflect deficits in coordination that are independent of any abnormalities in stretch related muscle activation. ${ }^{11}$

Spasms or sudden involuntary, often painful, movements are often included under the umbrella term of spasticity. However, physiologically these appear to be an independent entity. Spasms can be triggered not only by muscle stretch but also through a variety of peripheral, noxious, and visceral afferents. Spasms may be caused by disinhibited polysynaptic reflexes such as the flexor withdrawal reflex, ${ }^{12}$ or they may reflect abnormal activity within spinal cord circuits which have the effect of synchronising the discharge of motor neurones supplying multiple muscles. ${ }^{13}$

Spasticity and spasms are, however, only two of the symptoms of the UMN syndrome; other symptoms such as muscle weakness, decreased postural responses, and reduced dexterity all have an impact on an individual's function. These features may be independent of each other but it is often difficult to assess the relative contribution each has to reduction in function. For example, in hereditary spastic paraparesis it was often thought that spasticity was the main factor contributing to the abnormal gait, but if spasticity is effectively reduced-for example by intrathecal baclofen - there is often evidence of profound underlying weakness which is clearly contributing to the disability. ${ }^{14}$

\section{THE IMPACT OF SPASTICITY}

Spasticity can cause discomfort and stiffness, while spasms can be annoying and painful and may interfere with function. Physical activities such as walking, transferring, picking up objects, washing, dressing, and sexual activity can all be affected. Likewise the ongoing presence of spasticity and spasms can have an emotional impact on, for example, mood, self image, and motivation. Poorly managed spasticity can also be responsible for muscle shortening and the development of tendon and soft tissue contractures, which together with spasms can lead to compromised safety in lying and sitting..$^{15}$ Once contractures are present these are often very difficult to treat and can have major functional implications, including difficulties with personal hygiene or dressing, positioning, and at times the inability to sit, which may lead to restricted community mobility and social isolation. In addition, such difficulties can lead to the development of pressure sores, which may increase the

Abbreviations: FES, functional electrical stimulation; UMN, upper motor neurone 
severity of spasticity and spasms. A further problem-particularly in children with spasticity, which is often secondary to cerebral palsy-is the failure of normal muscle growth, resulting in torsion of long bones and consequent joint instability and degeneration. ${ }^{16}$ Early identification and intervention to treat spasticity and associated symptoms such as spasms can minimise the development of these long term secondary complications.

However, it must not be forgotten that spasticity can also be useful, perhaps allowing a person to stand or walk when weakness would not otherwise permit it. With these issues in mind it is imperative that management is always patient and function focused rather than aimed at the reduction of spasticity per se.

\section{EFFECTIVE MANAGEMENT MUST BE SEAMLESS AND MULTIDISCIPLINARY}

There is no agreed evidence based model available for the management of spasticity and much of what is done is based on a logical and pragmatic approach. A key component of management is the education of all involved, including the patient, family, carers, and health professionals. Continuity of care-particularly across the interfaces of primary and secondary care, involving community rehabilitation teams and care agencies-combined with documentation of the evolving impact of spasticity, is necessary to enable ongoing assessment of change and the appropriate choice and timing of any management intervention (fig 1). Whether the impact of spasticity on an individual is mild or severe, it is important that patients are knowledgeable about spasticity, its associated features, and how they can help themselves to manage and prevent symptoms. The trigger and aggravating factors detailed in fig 1 are particularly important as they can exacerbate spasticity and its associated features. Far too often pharmacological treatment is escalated before appropriate strategies to manage bladder and bowel function, skin integrity, soft tissue length, and positioning are instigated. Attention to these simple but essential areas is paramount at all stages of management.

It is often helpful to approach spasticity management according to the level of severity, including points at which intervention is essential to prevent secondary complications (table 1). However, regardless of the level of severity, it is always important to consider triggers and aggravating factors at each stage and determine whether the spasticity is predominantly focal or generalised (fig 1).

\section{MOVEMENT, EITHER PASSIVE OR ACTIVE, IS ESSENTIAL AT ALL STAGES OF MANAGEMENT}

Maintaining muscle length through passive or active exercise and stretching regimens including standing or splinting can be key to managing spasticity both in the short and the long term. Likewise, attention to posture and positioning, which may include the provision and regular review of seating systems, is paramount in managing severe spasticity. Doctors, physiotherapists, occupational therapists, and nurses across primary and secondary care can play key roles in working with the individual and their carers to assess the degree and impact of spasticity, identify goals of treatment, initiate referrals for specialist advice, implement management programmes, and monitor the effects of such interventions. ${ }^{17}$ Effective spasticity management requires clear communication and documentation between the individual and all the services involved in their care.

Short periods of inpatient rehabilitation can also be useful for individuals experiencing changes in their level of function; this may be increasing difficulty with walking or it could be the transition to the safe use of a wheelchair.

Functional electrical stimulation (FES) is an adjunct to physiotherapy that can be of benefit to selected individuals who are predominantly affected by UMN pathologies resulting in a dropped foot. In theory this should benefit both the neural (by reciprocal inhibition in antagonistic muscles) and non-neural (lengthening of soleus and gastrocnemius) aspects of spasticity. In a small randomised controlled trial in patients following stroke, the use of FES in combination with physiotherapy was statistically superior to physiotherapy alone. $^{18}$

\section{PHARMACOLOGICAL TREATMENT OF SPASTICITY}

Drug treatment can be used for generalised spasticity or targeted to focal problems, and can include agents such as botulinum toxin or intrathecal baclofen and phenol. Botulinum toxin is the most widely used treatment for focal spasticity. The effect of the toxin is to inhibit the release of acetylcholine at the neuromuscular junction. Although this blockade is permanent, the clinical effect of injecting botulinum toxin is reversible because of nerve sprouting and muscle reinnevation, leading to functional recovery of the muscle in a few months. ${ }^{19}$ It is essential that botulinum toxin injections be given in conjunction with physiotherapy to obtain the maximum benefit. The toxin is injected directly into the targeted muscle and takes 10 to 14 days to have a visible effect. As it is a reversible treatment it may have to be repeated after a few months. Despite randomised controlled trials showing that botulinum toxin is effective in reducing tone, ${ }^{20}$ there have been few that have shown an improvement in active function, although improvement in passive functions-ease of hygiene, dressing, and so on-has been demonstrated. A notable exception is the study by Brashear and colleagues, which demonstrated a reduction in spasticity in the wrist and fingers of patients following stroke, together with an improvement in their disability assessment scale. ${ }^{21}$ The lack of evidence of functional improvement in some studies may be related to poor trial design and the lack of function based outcome measures. $^{22}$

Local injection of phenol is an alternative option for focal spasticity management. This can be used alone or in combination with botulinum toxin, particularly in children, where small body size restricts the available treatment dose of botulinum toxin. Chemical neurolysis by phenol is irreversible and can be used at several sites. The most commonly used are medial popliteal blocks in the management of children with developing foot deformities, and obturator nerve blocks in ambulatory patients with scissoring gait or for improving sitting posture and ease of perineal hygiene. ${ }^{23}$

The oral agents most commonly used to treat spasticity are baclofen, tizanidine, benzodiazepines, dantrolene, and gabapentin. There is limited evidence of efficacy in clinical trials. The first four appear to be of similar benefit in multiple sclerosis, although diazepam is less well tolerated than the others. Gabapentin has only been studied in a few short term trials (two to six days). ${ }^{24}$ With the exception of tizanidine and dantrolene, these drugs act by potentiating the action of the inhibitory neurotransmitter gamma amino butyric acid (GABA). Tizanidine, however, is predominantly an $\alpha_{2}$ agonist and thus decreases presynaptic activity of the excitatory interneurones. Dantrolene is the only antispasticity treatment that acts primarily on muscle. Through inhibiting calcium release from the sarcoplasmic reticulum it decreases the excitation-coupling reaction involved in muscle contraction.

Unfortunately all drug treatments for spasticity can have side effects, the commonest of which are drowsiness 


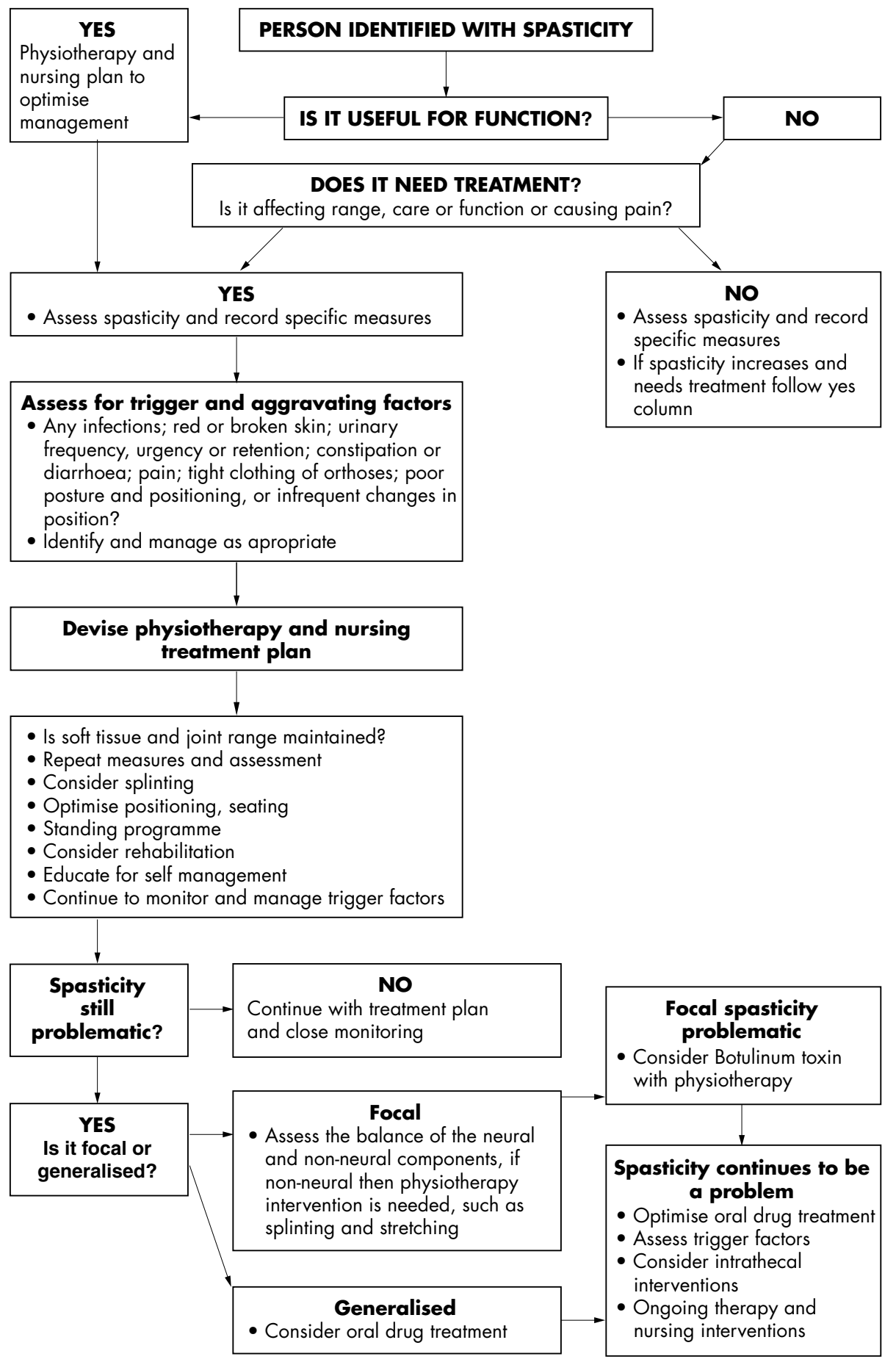

Figure 1 Algorithm for management of spasticity.

and weakness. Of course it is important to recognise that a perceived increase in weakness may actually be a result of unmasking the impairment by removing tone which was functionally useful. To optimise the effects of oral drug treatment it is important to identify appropriate dosage regimens. For instance, if getting out of bed is difficult, the drugs should be left next to the bed and taken immediately on waking, preferably 10 to 20 minutes before getting up. If noctur- nal spasms are a particular problem, then increasing night-time doses can be useful.

If oral drug treatment is inadequate at controlling lower limb spasticity or is not tolerated, then intrathecal delivery of baclofen should be considered. The concentration of GABA receptors in the lumbar spinal cord allows very small dosages of baclofen to be effective without causing any systemic side effects. The programmable pump is implanted into the abdomen, from where a catheter conveys the baclofen into the intrathecal space. This is obviously an invasive and relatively expensive treatment which requires careful selection of patients, a trial using a bolus dose of baclofen through a lumbar puncture needle, and significant commitment from patient, not only during the trial and implant phase but also for the ongoing maintenance of regular refills and replacements. 
Table 1 Spasticity management according to level of severity

Mild spasticity

- Clonus or mild increase in tone

- No or minimal loss of range

- Mild spasms; generally not problematic or affecting function but annoying or inconvenient

Moderate spasticity

- Loss of range of movement and possible contracture

- Walking is often effortful, may require aid or wheelchair

- Difficulty releasing grip or in hand hygiene

- Minor adaptations required for position in lying; t-roll, wedge, pillows, lumbar roll

Emphasis on self management, education, and how to seek help to prevent secondary complications

- Highlight potential trigger factors

- Education of secondary complication

- Stretches: neurophysiotherapist identifies vulnerable areas and recommends specific, active, and regular stretches

- Discuss ways of maintaining active movement and modify patterns of movement to minimise increasing spasticity

- Low dose drug treatment targeted at problematic times of the day, with ongoing review and evaluation, maybe beneficial

Emphasis on early identification and treatment of trigger factors, review of self management knowledge, and liaison with team members involved throughout the different health care sectors; maximise the use of oral drugs and consider the use of botulinum toxin if focal

- Identify trigger factors and treat as appropriate

- Targeted neurophysiotherapy; active stretching, exercise and standing programmes; consider splinting

- Maximise available activity to have a positive impact on function and establish extent to which spasticity and spasms are used to enable effective movement; consider rehabilitation

- Accurately document assessments and treatments to enable ongoing evaluation of intervention

- Maximise oral drugs and consider if botulinum toxin could be helpful for focal spasticity

- Consider referral to specialist service

Severe spasticity

Emphasis on maximising use of oral and focal drug treatments while considering use of intrathecal baclofen or phenol; review patient's, carers' and health team management strategies

- Identify trigger factors and treat as appropriate

- Accurately document assessments and treatments to enable ongoing evaluation of intervention

- Assess effectiveness of current treatment strategies, modify as appropriate, and consider intrathecal drugs

- If intrathecal drugs are used, review need to reassess seating, transfers, and therapy input; consider rehabilitation

- If treatments are or become ineffective, review management strategies including possibility of surgery

- Identify trigger factors and treat as appropriate

- Accurately document assessments and treatments to enable ongoing evaluation of intervention

- Assess effectiveness of current treatment strategies, modify as appropriate, and consider intrathecal drugs

- If intrathecal drugs are used, review need to reassess seating, transfers, and therapy input; consider rehabilitation

- If treatments are or become ineffective, review management strategies including possibility of surgery
Occasionally the measures outlined above are not sufficient to manage severe spasticity causing contractures and preventing carers from seating or hoisting the patient safely, and such patients may be considered for intrathecal phenol treatment. As phenol is a destructive agent which indiscriminately damages motor and sensory nerves, it is reserved for those individuals who do not have any functional movement in the legs, who have lost bladder and bowel function, and who have impaired sensation to the legs. Phenol is injected intrathecally and is prepared in glycerin, which renders it hyperbaric and viscous, thus limiting its spread. Intrathecal phenol can be an effective treatment which, though it requires expert administration, does not have the long term maintenance or cost issues that go with intrathecal baclofen treatment. The effect of a single injection often lasts many months and can be repeated if necessary. ${ }^{25}$

\section{MONITORING SPASTICITY}

It is clearly helpful to adopt a management approach that is linked to the severity of the spasticity. However, this implies an accurate and reliable assess- ment of severity, which is in itself a challenging area. Research is ongoing into different assessment strategies, including gait analysis and biomechanical, neurophysiological, and clinical measurements. The first and best known scale for measuring the degree of spasticity was the Ashworth scale, first developed in 1964 for use in a multiple sclerosis therapeutic trial. ${ }^{26}$ However, this is a single item scale with poor validity, reliability, and responsiveness. ${ }^{27}$ Neither is the Ashworth grade influenced by associated features including pain or spasms, or by impact on function; all of which are important issues for the patient. Other clinical scales have been used, including clinician visual analogue scales or self report scales for spasm severity and frequency, though none has been shown to be reliable. ${ }^{28}{ }^{29}$ There is thus still a need for development of a valid, responsive, and reliable scale to assess both the severity and the functional impact of spasticity and its associated features. ${ }^{30}$ In addition to clinical scales, more complex techniques including pendulum tests or gait analysis can be employed, as well as simple goniometers allowing ranges of movement in relevant joints to be recorded and monitored. ${ }^{31}$ In our experience several measures are necessary to complete a thorough evaluation of the severity and impact of an individual's spasticity. Our battery includes the Ashworth scale, range measures at rest, active movement and full stretch using a goniometer at joints, and measuring the distance between the knees. We also use subjective severity scores of stiffness, clonus, spasms, pain, and overall comfort, a sitting tolerance score, and recordings of a timed 10 metre walk and frequency of falls. Functional difficulties are outlined in a patient tailored goal of treatment form.

\section{CONCLUSIONS}

Spasticity is one of the components of the UMN syndrome but should not be considered in isolation when it comes to management strategies. It is essential that management targets function and is always patient focused rather than aimed at reducing the degree of spasticity. A management strategy is required, such as that prepared by the Multiple Sclerosis Council for their clinical practice guidelines, ${ }^{32}$ which incorporates an understanding of spasticity in the context of the UMN syndrome with its 
associated features, aggravating factors, and trigger factors. This is of paramount importance for the patient, family, carers, and health teams. This allows self management strategies to be employed, but also the knowledge essential to identify when further treatment strategies are needed to prevent secondary complications. There is a wide range of physical and pharmacological treatments available which, if used in a timely and appropriate fashion, can be very useful at reducing pain and discomfort without compromising function.

J Neurol Neurosurg Psychiatry 2005;76:459-463.

doi: $10.1136 /$ innp.2004.035972

\section{Authors' affiliations}

L Jarrett, L Lockley, V L Stevenson, National

Hospital for Neurology and Neurosurgery,

Queen Square, London WCl, UK

A J Thompson, J Marsden, Institute of

Neurology, Queen Square

Correspondence to: Professor Alan J Thompson, Institute of Neurology, Queen Square, London WCIN 3BG, UK; a.thompson@ion.ucl.ac.uk

Received 1 July 2004

In revised form 27 October 2004

Accepted 28 October 2004

Competing interests: none declared

\section{REFERENCES}

1 Brown P. The pathophysiology of spasticity. J Neurol Neurosurg Psychiatry 1991;238:131-9.

2 Burke D. Spasticity as an adaptation to pyramidal tract injury. Adv Neurol 1988:47:401-23.

3 Wilson LR, Gandevia SC, Inglis JT, et al. Muscle spindle activity in the affected upper limb after a unilatreral stroke. Brain 1999;122:2079-88.
4 Aymard C, Katz R, Lafitte C, et al. Presynaptic inhibition and homosynaptic depression. A comparison between lower and uppper limbs in normal human subjects and patients with hemiplegia. Brain 2000;123:1688-702.

5 Zijdewind I, Thomas CK. Spontaneous motor unit behavior in human thenar muscles after spinal cord injury. Muscle Nerve $2001 ; 24: 952-62$.

6 Zijdewind I, Thomas CK. Motor unit firing during and after voluntary contractions of human thenar muscles weakened by spinal cord injury. I Neurophysiol 2003;89:2065-71.

7 Bennett DJ, Li Y, Harvey PJ, et al. Evidence for plateau potentials in tail motoneurons of awake chronic spinal rats with spasticity. J Neurophysiol 2001;86:1972-82.

8 Nickolls P, Collins DF, Gorman RB, et al. Forces consistent with plateau-like behaviour of spinal neurons evoked in patients with spinal cord injuries. Brain 2004; 127:660-70.

9 Dietz V. Spastic movement disorder: what is the impact of research on clinical practice? J Neurol Neurosurg Psychiatry 2003;74:820-6.

10 Dewald JP, Pope PS, Given JD, et al. Abnormal muscle coactivation patterns during isometric torque generation at the elbow and shoulder in hemiparetic subjects. Brain 1995; 118:495-510.

11 Canning CG, Ada L, O'Dwyer NJ. Abnormal muscle activation characteristics associated with loss of dexterity after stroke. J Neurol Sci 2000;176:45-56

12 Schmit BD, Benz EN, Rymer WZ. Reflex mechanisms for motor impairment in spinal cord injury. Adv Exp Med Biol 2002;508:315-23.

13 Norton JA, Marsden JF, Day BL. Spinally generated electromyographic oscillations and spasms in a low-thoracic complete paraplegic. Mov Disord 2003;18:101-6.

14 Richardson D, Thompson AJ. Management of spasticity in hereditary spastic paraplegia. Physiother Res Int 1999;4:68-76.

15 Jarrett $L$. The role of the nurse in the management of spasticity. Nurs Resident Care 2004;3:116-19.

16 Koman LA, Smith BP, Shilt JS. Cerebral palsy Lancet 2004;363:1619-31.

17 Richardson D. Physical therapy in spasticity. Eur J Neurol 2002;9:17-22.

18 Burridge J, Taylor P, Hagan S, et al. The effects of common peroneal stimulation on the effort and speed of walking: a randomised controlled clinical trial with chronic hemiplegic patients. Clin Rehabil 1997; 11:201-10.

19 Davis EC, Barnes MP. Botulinum toxin and spasticity. J Neurol Neurosurg Psychiatry 2000:69:143-9.

20 Richardson D, Sheean G, Werring D, et al. Evaluating the role of botulinum toxin in the management of focal hypertonia in adults. J Neurol Neurosurg Psychiatry 2000;69:499-506.

21 Brashear A, Gordon MF, Elovic E, et al. Botox Post-Stroke Spasticity Study Group. Intramuscular injection of botulinum toxin for the treatment of wrist and finger spasticity after a stroke. N Engl J Med 2002;347:395-400.

22 Sheean GL. Botulinum treatment of spasticity: why is it so difficult to show a functional benefit? Curr Opin Neurol 2001;14:771-6.

23 Barnes MP. Local treatment of Spasticity. Baillieres Clin Neurol 1993;2:55-71.

24 Beard S, Hunn A, Wright J. Treatments for spasticity and pain in multiple sclerosis: a systematic review. Health Technol Assess 2003;7:1-111.

25 Jarrett L, Nandi P, Thompson AJ. Managing severe lower limb spasticity in multiple sclerosis: does intrathecal phenol have a role? J Neurol Neurosurg Psychiatry 2002;73:705-9.

26 Ashworth B. Preliminary trial of carisoprodal in multiple sclerosis. Practitioner 1964;192:540-2.

27 Hobart J. Rating scales for neurologists. J Neurol Neurosurg Psychiatry 2003;74/suppl IV):iv705-9.

28 Pomeroy VM, Dean D, Sykes L, et al. The unreliability of clinical measures of muscle tone: the implications for stroke therapy. Age Ageing 2000;29:229-33.

29 Riazi A, Fox P, Vickery J, et al. Developing a patient-based measure of the impact of spasticity in multiple sclerosis. Multiple Sclerosis 2003;9:S151.

30 Priebe MM, Sherwood AM, Thornby Jl, et al. Clinical assessment of spasticity in spinal cord injury: a multidimensional problem. Arch Phys Med Rehabil 1996;77:713-16.

31 Johnson GR. Outcome measures of spasticity. Eur J Neurol 2002;9(suppl 1):10-16.

32 Multiple Sclerosis Council for Clinical Practice Guidelines. Spasticity management in multiple sclerosis: evidence based management strategies for spasticity treatment in multiple sclerosis. Paralyzed Veterans of America/ Consortium of Multiple Sclerosis Centres, 2003:27pp.

\section{Cerebral revascularisation: where are we now?}

\section{P J Kirkpatrick, I Ng}

\section{Outcomes may be improved by standardising measurement of $\mathrm{CBF}$ and the surgical approach and centralising expertise}

E nthusiasm for delivering an alternative blood supply to the brain by surgical means has waxed and waned for over three decades. In situations where a major cerebral vessel is sacrificed for removal of macroscopic pathology (such as a skull base tumour or giant intracranial aneurysm), the need to replace lost cerebral blood flow $(\mathrm{CBF})$ is obvious and often required as part of a staged surgical procedure. ${ }^{12}$ In such cases, the need and type of surgical bypass graft (high or low flow) is dictated by the presence or absence of adequate collateral vascular pathways, the design of the circle of Willis, and any extracranial to intracranial (EC-IC) vascular connections. ${ }^{3}$ Detailed cranial angiography and observation of the clinical and physiological responses to temporary test occlusion of parent vessels provide the relevant information. When reliably practised, daunting cervical and cranial pathologies can be approached confidently with acceptable morbidity. ${ }^{4}$ Although simple in principle, techniques for assessing the need for surgical bypass procedures are not always practised resulting in incomplete treatments, and/or high surgical morbidity from cerebral strokes. Centralisation of expertise and adoption of a more appropriate referral of difficult pathology will serve to address the variation in practice for such cases. 


\section{CEREBRAL REVASCULARISATION IN CHRONIC VASCULAR OCCLUSION}

A far more intense debate surrounds the indications for cerebral revascularisation in the treatment of chronic vascular occlusive disease. Extracranial atheromatous degenerative vascular disease is by far the commonest pathological entity falling into this group, ${ }^{5}{ }^{6}$ although intracranial atheroma and chronic inflammatory disease (Moya Moya) is significant in Eastern communities. Patients usually present with neurological events leading to investigation and identification of occlusive disease. However, unlike stenotic disease, occluded vessels do not attract a high risk of future embolic events. ${ }^{5-7}$ Most patients achieve normal or near normal CBF by means of the aforementioned collaterals. Only a minority of those who survive the occlusion without an immediate major stroke exist in a chronic state of low CBF. Accurate neurological evaluation of the presenting symptoms (retinal $v$ cerebral) is clearly important in determining the risk of further clinical events. ${ }^{8}$ However, from a clinical and pathophysiological standpoint, these patients should be considered for CBF assessment and cerebral bypass surgery-not for CBF replacement but for CBF augmentation. ${ }^{9-11}$

\section{WHY THE CONTINUED DEBATE?}

Since the first description of an EC-IC bypass procedure by Yasargil, ${ }^{12}$ much harm has been done in the wide propagation of the procedure without resort to a sound physiological or clinical substrate, upon which to base the procedure. Most patients presenting with symptomatic carotid occlusive disease accommodate the pathology well; hence, most do not need a cerebral bypass. Undiscerning surgical practice raised medical concerns, the result was the controversial multicentre Cooperative Study (EC-IC Bypass Study) that failed to observe any benefit from the bypass procedure. ${ }^{13}$ The widespread surgical practice of EC-IC bypass collapsed, largely as a result of withdrawal of the funding mechanisms supporting the operation. Those who were more thoughtful in the application of EC-IC bypass procedures dissented. ${ }^{14-16}$ Why was there no selection of patients more suitable for the procedure, particularly those who were clinically symptomatic of cerebral hypoperfusion? Why were so many patients excluded from the study? Was the study cohort largely represented by patients in whom there was uncertainty? Indeed, was the uncertainty principle helpful in this regard? The Cooperative Study addressed only a part of contemporary surgical practice and failed to observe the basic physiological and clinical indications for the operation. A negative result was inevitable.

Some clinical doors remained open. Pioneers of selective EC-IC procedures have accumulated supporting and compelling information addressing a cohort of patients who attract high risks for future stroke, risks that reflect a chronic state of low CBF..$^{9-11}{ }^{17-21}$ Of the variety of techniques used to measure CBF, observations of baseline values are generally unhelpful. Activated studies measuring relative increases in $\mathrm{CBF}$ have greater predictive values for identifying those at risk. ${ }^{17-21}$ Hence a number of cerebrovascular reactivity indices have evolved which, although different, point in the same direction-impaired cerebrovascular reactivity is a risk factor for future strokes. Those with negative reactivity on acetazolamide activated xenon computed tomography (CT) have a 12-fold increase in incidence of stroke, ${ }^{22}$ and those with an increased oxygen extraction fraction indicating exhausted cerebrovascular reserve (measured using positron emission tomography (PET)) have an increased stroke risk of $30 \%{ }^{23}{ }^{24}$ Likewise, severely impaired CBF reactivity to carbon dioxide (measured using transcranial Doppler flowmetry) also increases the risk by approximately $30 \% .{ }^{18}{ }^{25}$ Two ongoing randomised trials have adopted different physiological criteria upon which to base surgery. The North American Carotid Occlusion Surgery Study $(\text { COSS })^{26}$ is using PET derived oxygen extraction fraction, whereas the Japanese Extracranial to Intracranial Bypass Trial $(\mathrm{JET})^{27}$ is using acetazolamide CBF activation measured with either single photon emission CT or xenon CT imaging. JET has already demonstrated significant short term gains for the surgical group, and the imaging modalities adopted have the obvious advantage of far wider clinical availability than PET derived indices.

The key questions remaining are:

- What is the threshold for cerebral perfusion that defines the patient at risk?

- Which technique for CBF measurement should be used?

- Which cerebrovascular indices have the greatest sensitivity for prediction of stroke?

- What are the absolute benefits of surgery?

- What surgical procedure is best?

- What rates for poor surgical outcome can be accepted?
Without a universally accepted measurement for $\mathrm{CBF}$, and without adopting a standardised surgical approach, gains for cerebral revascularisation will be dilute, and clinical benefits hard to identify. Similar concerns have already muddied the waters for treatment of other cerebrovascular conditions. Thus in the same way that carotid endarterectomy needs to meet vigorously defined surgical standards, ${ }^{28}$ the same will be true for EC-IC bypass. Indeed, more so in the face of a technically far more demanding operation. Low flow bypasses can normalise an abnormal oxygen extraction fraction and regional $\mathrm{CBF}^{29-33}$ and reverse neurocognitive deficits. ${ }^{34}$ Hence the technically more demanding high flow bypass (using a saphenous vein or radial artery graft) seems unnecessary when considering an operation to provide CBF augmentation. Less conventional methods for cerebral revascularisation, such as the use of vascular tissues applied directly to the cerebral cortex, require similar physiological and clinical scrutiny before their use can be justified.

\section{CONCLUSION}

With relatively small numbers of suitable cases, centralisation of expertise would be important for achieving a measure of surgical quality control. Once broad criteria for offering cerebral revascularisation have been accepted and parallel surgical standards defined and met, the thresholds for surgery in individual centres can be developed further. Adoption of a multidisciplinary team approach assists in accurate patient assessment and selection and management of vascular risk factors, and provides an environment for testing novel applications for revascularisation surgery.

\section{J Neurol Neurosurg Psychiatry}

2005;76:463-465.

doi: 10.1136/jnnp.2004.035998

\section{Authors' affiliations}

P J Kirkpatrick, University Department of

Neurosurgery, Addenbrooke's Hospital,

Cambridge, UK

I Ng, Department of Neurosurgery (TTSH Campus), National Neuroscience Institute, Singapore

Correspondence to: Mr P J Kirkpatrick, University Department of Neurosurgery, Block A Level 4, Addenbrooke's Hospital, Cambridge CB2 2QQ, UK; pjk21@medschl.cam.ac.uk

Competing interests: none declared

\section{REFERENCES}

1 Jafar JJ, Russell SM, Woo HH. Treatment of giant intracranial aneurysms with saphenous vein extracranial-intracranial bypass grafting: 
indications, operative technique and results in 29 patients. Neurosurgery 2002;51:138-46.

2 Sekhar LN, Bucur SD, Bank WO, et al. Venous and arterial bypass grafts for difficult tumours, aneurysms, and occlusive vascular lesions: evolution of surgical treatment and improved graft results. Neurosurgery 1999:44:1207-24.

3 Leibeskind DS. Collateral circulation. Stroke 2003;34:2279-84

4 Tarr RW, Jungries CA, Horton JA, et al. Complications of pre-operative test occlusion of the internal carotid arteries: Experience in 300 cases. Skull Base Surg 1991;1:240-4.

5 Flaherrty ML, Flemming KD, McClelland R, et al. Population- based study of symptomatic internal carotid artery occlusion. Stroke

2004;35:349-52.

6 Pessin MS, Duncan GW, Mohr JP, et al. Clinical and angiographic features of carotid transient ischaemic attacks. N Engl J Med 1977;296:358-62

7 Hankey GJ, Slattery JM, Warlow CP. The prognosis of hospital-referred transient ischaemic attacks. J Neurol Neurosurg Psychiatry 1991;54:793-802

8 Klijn CJ, Kappelle $\sqcup$, van Huffelen AC, et al. Recurrent ischemia in symptomatic carotid occlusion: prognostic value of hemodynamic factors. Neurology 2000;26:1806-12.

9 Grubb RL, Derdeyn CP, Fritsch SM, et al. Importance of hemodynamic factors in the prognosis of symptomatic carotid occlusion. JAMA 1998;280:1055-60.

10 Yamauchi H, Fukuyama H, Nagahama Y, et al Evidence of misery perfusion and risk for recurrent stroke in major cerebral arterial occlusive diseases from PET. J Neurol Neurosurg Psychiatry 1996;61:18-25.

11 Durham SR, Pentheny SL, Johnson DW. Increased stroke risk predicted by compromised cerebral blood flow reactivity. $J$ Neurosurg 1993:79:483-9.

12 Yasargil MG. Anastomosis between the superficial temporal artery and a branch of the middle cerebral artery. In: Yasargil MG, ed. Microsurgery applied to neurosurgery. Stuttgart: Georg Thieme, 1969:105-15.

13 EC/IC Bypass Study Group. Failure of extracranial-intracranial arterial bypass to reduce the incidence of ischemic stroke. N Engl J Med 1985;313:1191-2000.

14 Ausman IA, Diaz FG. Critique of the extracranialintracranial bypass study. Surg Neurol 1986;26:218-21.

15 Awad IA, Spetzler RF. Extracranial-intracranial bypass surgery: a critical analysis in light of the International Cooperative Study. Neurosurgery 1986; 19:655-64.

16 Sundt TM. Was the international randomized trial of extracranial-intracranial arterial bypass representative of the population at risk? N Engl J Med 1987;316 814-16.

17 Gibbs JM, Leenders KL, Wise RJS, et al. Evaluation of cerebral perfusion pressure reserve in patients with carotid- artery occlusion. Lancet 1984; 1:310-14.

18 Powers WJ, Tempel LW, Grubb RL Jr. Influence of cerebral hemodynamics on stroke: one year follow-up of 30 medically treated patients. Ann Neurol 1989;25:325-30

19 Kleiser B, Widder B. Course of carotid occlusions with impaired cerebrovascular reactivity. Stroke 1992;23:171-4.

20 Ogasawara K, Ogawa A, Terasaki K, et al Use of cerebrovascular reactivity in patients with symptomatic major cerebral artery occlusion to predict 5-year outcome: comparison of xenon-133 and iodine123-IMP single-photon emission computed tomography. J Cereb Blood Flow Metab 2002;22:1142-8.

21 Kuroda S, Houkin K, Kamiyama H, et al. Long term prognosis of medically treated patients with internal carotid or middle cerebral artery occlusion: can acetazolamide test predict it? Stroke 2001:32:21 10-16.

22 Yonas H, Smith HA, Durham SR, et al. Increased stroke risk predicted by compromised cerebral blood flow reactivity. J Neurosurg 1993:79:483-9.

23 Derdeyn CP, Videen TO, Simmons NR, et al. Count-based PET method for predicting ischemic stroke in patients with symptomatic carotid arterial occlusion. Radiology 1999:212:499-506.

24 Yamauchi $\mathrm{H}$, Fukuyama $\mathrm{H}, \mathrm{Nagahama} Y$, et al. Significance of increased oxygen extraction fraction in five-year prognosis of major cerebral arterial occlusive diseases. J Nucl Med 1999:40:1992-8.

25 Markus H, Cullinane M. Severely impaired cerebrovascular reactivity predicts stroke and TIA risk in patients with carotid artery stenosis and occlusion. Brain 2001;124 457-67.

26 Adams HP, Powers WJ, Grubb RL, et al. Preview of a new trial of extracranial to intracranial arterial anastomosis: the Carotid Occlusion Surgery Study. Neurosurg Clin North Am 2001;12:613-24

27 Ogawa A. Cerebrovascular imaging and bypass surgery. Presented at the 1 st Asian Pacific Stroke Conference against stroke. Hong Kong, 2004

28 Executive Committee for the Asymptomatic Carotid Atherosclerosis Study. Endarterectomy for asymptomatic carotid artery stenosis. JAMA 1995;273:1421-28.

29 Schmideck P, Piepgras A, Leinsinger G, et al. Improvement of cerebrovascular reserve capacity by EC-IC bypass surgery in patients with ICA occlusion and hemodynamic cerebral ischemia. 1994;81:236-44.

30 Gibbs JM, Wise RJS, Thomas DJ, et al. Cerebral hemodynamic changes after extracranialintracranial bypass surgery. $J$ Neurol Neurosurg Psychiatry 1987;50:140-50.

31 Powers WJ, Martin WRW, Herscovitch P, et al. Extracranial-intracranial bypass surgery: hemodynamic and metabolic effects. Neurology 1998:34:1168-74.

32 Samson Y, Baron JC, Bousser MG, et al. Effects of extracranial-intracranial arterial bypass on cerebral blood flow and oxygen metabolism in humans. Stroke 1985;16:609-16

33 Yamashita T, Kashiwagi S, Nakano S, et al. The effects of EC-IC bypass surgery on resting cerebral blood flow and cerebrovascular reserve capacity studied with stable $\mathrm{Xe}-\mathrm{CT}$ and acetazolamide test. Neuroradiology 1991;33:217-22

34 Sasoh M, Ogasawara K, Kuroda K, et al. Effects of EC-IC bypass on cognitive impairment in patients with hemodynamic cerebral ischemia Surg Neurol 2003;59:455-60. emphasis of "motor re-learning" is on context specific functional training using principles derived from motor learning theory, while that of the Bobath concept is on observational analysis and facilitation of normal movement using principles derived from neurodevelopmental and neurophysiological theory.

van Vliet and colleagues have previously shown that therapy using these two approaches differs, for example in the degree and type of feedback provided and extent to which everyday objects are used during the treatment session. ${ }^{3}$ Their single blind randomised controlled trial, ${ }^{2}$ which admitted 120 patients within two weeks of stroke, has now shown no differences in sensorymotor impairments or functional outcome between the two interventions up to six months after treatment onset. Whether these findings are the result of 
similarities between the two approaches far outweighing their differences or for other reasons is unclear and worthy of further exploration.

\section{THE NEED TO DEFINE THE INTERVENTION}

As van Vliet and colleagues observed, ${ }^{2}$ interpreting a study of this sort is made difficult by the variability between studies, not only in the content of the intervention itself but also in the context in which the intervention occurs. Physiotherapy interventions of this sort are complex and are "made up of various interconnecting parts." ${ }^{\prime 4}$ If further studies of this sort are to be done, it will be important to define the practice protocols for each intervention rigorously, in particular how the type and difficulty of tasks used within a treatment session are chosen, and the ways in which treatment is progressed; observational analysis of the different interventions is an important step in this direction. ${ }^{13}$

Detailed definition of intervention protocols would facilitate study reproduction and comparison, identify the similarities and differences between interventions, and enable investigation of the effects of other variables such as the onset and intensity of treatment. Intensity of treatment is not necessarily the same as the duration of treatment, as an equivalence of treatment time between interventions may not guarantee an equivalence of treatment intensity. Future protocols should not only aim to define the components of a treatment session but also to define how the practice session is scheduled as this may be important in optimising the learning of a motor task. Factors such the type and frequency of feedback and whether different tasks are practised consecutively or their practice order randomised may affect the rate and extent to which a task is learnt, as well as the extent to which it may generalise to novel environmental situations both in health and after stroke. ${ }^{56}$
Given the wide differences in the severity and types of functional deficit after stroke, and the ever evolving ethos of the different approaches, formulating such protocols will be difficult. In addition, the other interventions and activities that occur outside the daily $\sim 30$ minute therapy session may have a significant impact, to the extent that differences in the efficacy of one physiotherapy approach over another may be swamped by the similarities in activities that occur for the remaining $\sim 98 \%$ of the time. How studies can avoid this "noise" needs exploration.

\section{THE NEED TO ASSESS COMPONENTS OF AN INTERVENTION}

The stroke population studied by van Vliet was heterogeneous, reflecting that seen in clinical practice. Such a holistic approach may overlook some of the intricacies of an approach. The different components of each approach are largely based on the theoretical construct with which it is underpinned; the effects of each component have been explored very little even in small proof of principle studies. The efficacy of different approaches and their components may be dependent on stroke pathology and the presenting impairments. Furthermore, a more detailed biomechanical assessment may reveal differences in the effects of two approaches. Although such differences may not be regarded as important unless they are revealed in snapshots of functional outcome, differences in movement patterns, and in particular movement efficiency, may be important for the ability to perform a task repetitively, as is required for everyday functioning, or for the gradual acquisition of a skill over a protracted period.

Thus two research strands are required to improve our current physiotherapy practice: first a top down, holistic approach, in which the practice content, schedule, and intensity are defined within the context of an overall standardised treatment package; second, a bottom up, reductionist approach where individual components of an intervention are assessed on specific patient populations in a hypothesis driven manner. As highlighted by van Vliet et al, ${ }^{2}$ one disadvantage of a reductionist approach is that it neglects potentially important interactions between treatment techniques, emphasising the need for a combined approach

J Neurol Neurosurg Psychiatry 2005;76:465-466

doi: 10.1136/jnnp.2004.053827

\section{Authors' affiliations}

J Marsden, Sobell Department of Movement

Neurosciences, Institute of Neurology, Queen Square, London WC1, UK

R Greenwood, Regional Neurological Rehabilitation Unit, Homerton Hospital, London E9, and Acute Brain Injury Service, National Hospital for Neurology and Neurosurgery, Queen Square, London WC1, UK

Correspondence to: Dr R J Greenwood, RNRU, Homerton University Hospital, Homerton Row, London E9 6SR, UK; richard.greenwood@ homerton.nhs.uk

Competing interests: none declared

\section{REFERENCES}

1 Lennon S, Baxter D, Ashburn A. Physiotherapy based on the Bobath concept in stroke rehabilitation: a survey within the UK. Disabil Rehabil 2001 23:254-62.

2 van Vliet PM, Lincoln NB, Foxall AD. Comparison of Bobath-based and movement science based treatment for stroke: a randomised controlled trial. J Neurol Neurosurg Psychiatry 2005;75:503-8

3 van Vliet PM, Lincoln NB, Robinson E. Comparison of the content of two physiotherapy approaches for stroke. Clin Rehabil 2000;15:398-414.

4 Campbell M, Fitzpatrick R, Haines A, et al. Speigelhalter D, Tyrer P. Framework for design and evaluation of complex interventions to improve health. BMJ 2000;321:694-6.

5 Hanlon RE. Motor learning following unilateral stroke. Arch Phys Med Rehabil 1996;77:81 1-15.

6 Boyd LA, Winstein CJ. Providing explicit information disrupts implicit motor learning after basal ganglia stroke. Learning Memory 2004; 11:388-96. 


\section{A failure to communicate: patients with cerebral aneurysms and vascular neurosurgeons}

D Leys, J-P Lejeune, J-P Pruvo
Many patients have an inaccurate understanding of their treatment plan

\footnotetext{
$p$
} $\mathrm{n}$ the paper by King et al (this issue, pp 550-4), ${ }^{1}$ an analysis of the communication between neurosurgeons and their patients with unruptured aneurysms was conducted immediately after an outpatient appointment in a neurosurgery clinic. Many patients had an inaccurate understanding of their treatment plan, and an exaggerated sense of the risks of treatments and of the disease. Despite methodological limitations discussed by the authors, the results of this study are consistent with those found in other specialties.

Communication is of special importance whenever there is a choice between two therapeutic options-that is, when the decision depends mainly on the patient's preference. Practitioners should be sure that their information was correctly understood. This holds true especially when the event we want to prevent is potentially devastating, the therapeutic options invasive and risky, leading to irreversible changes, and the risk of spontaneous occurrence of this event is low. Beside unruptured aneurysms, this situation is also met, for example, in carotid surgery for asymptomatic stenosis, interventional radiology for arteriovenous malformations that have never bled, asymptomatic cavernomas, and asymptomatic meningiomas.

The quality of the information is crucial because the relation between doctors and patients has changed: years ago, doctors used to decide what they felt to be the best option for their patient. Nowadays, when there is no emergency they have to explain the various options and their advantages and disadvantages, and finally the patient decides. This is an improvement for the quality of care, but unfortunately the last step is still missing: it is necessary to check carefully the patient's level of knowledge before they take a decision, otherwise their decision may be inappropriate.

To increase the quality of the information, seven actions can be recommended: (1) encourage randomised clinical trials for any clinical situation frequent enough, where there is uncertainty, to provide patients with more reliable information; (2) never ask the patient to take a decision immediately when there is no emergency; (3) organise a visit with a practitioner who is not personally involved in the techniques and has neither preference nor reluctance for one or other technique, for instance a neurologist; (4) explain the issue to the general practitioner and involve them in the discussion; (5) check whether patients have really understood the discussion, especially when they have a low level of education; (6) bear in mind that patients are always more pessimistic for their own health and exaggerate the risks of the disease (possibly leading to an inappropriate wish to be treated) and of the treatments (possibly leading to an inappropriate refusal of invasive options); (7) bear in mind that urgent decisions reduce the level of anxiety in the practitioner but are major obstacles to a multidisciplinary approach, which is the best guarantee of providing balanced information.

J Neurol Neurosurg Psychiatry 2005;76:467. doi: 10.1136/jnnp.2004.054460

\section{Authors' affiliations}

D Leys, J-P Lejeune, J-P Pruvo, University of Lille, Service de Neurologie B, Hôpital Roger Salengro, Rue Emile Laine, Lille 59037, France

Correspondence to: Professor Didier Leys, dleys@nordnet.fr

Competing interests: none declared

\section{REFERENCE}

1 King JT, Yonas $\mathrm{H}$, Horowitz $\mathrm{MB}$, et al. A failure to communicate: patients with cerebral aneurysms and vascular neurosurgeons. J Neurol Neurosurg Psychiatry 2005;88:550-4.
Malaria

\section{Severe malaria: still counting the costs}

\section{G L Birbeck, T E Taylor}

\section{The cost of severe malaria}

$\mathrm{T}$ he burden of malaria in the developing world has largely been measured in terms of childhood mortality. Annually, 300-500 million infections occur resulting in 1 million deaths; these account for $20 \%$ of childhood mortality in malaria endemic regions. ${ }^{1}$ An article by Carter et al (this issue, $p p$ 476-81) provides evidence that the burden of malaria includes chronic neurological consequences among survivors. ${ }^{2}$

The possibility that survivors of severe malaria suffer from neurological sequelae such as cognitive deficits or epilepsy has been suggested. ${ }^{3}$ Certainly, gross deficits at hospital discharge among a small proportion of survivors have been reported, but until recently, little systematic data on long term outcomes of severe malaria were available. Previous studies have suffered from loss to follow-up, short duration of follow-up, the lack of a comparison population, 
and evaluations limited to a bedside assessment.

The study by Carter et al utilised the Kilifi District Hospital database to identify survivors of severe malaria in rural Kenya. Subjects had experienced either cerebral malaria (CM) (peripheral parasitemia plus $\geqslant 4$ hours of coma) or malaria with complicated seizures (M/S) (peripheral parasitemia plus multiple, prolonged, or focal seizures). A comparison group comprised of age matched children was identified from a census of the catchment region. Follow-up occurred up to 9 years after the infection. All study subjects underwent neurologic assessments, including standardised cognitive testing validated in this population. The findings were striking. Impairments were identified in $24 \%$ of $\mathrm{CM}$ and $\mathrm{M} / \mathrm{S}$ children compared with $10 \%$ of the comparison population. Among impaired children, multiple impairments were also more common among CM (42\%) and M/S (30\%) than the comparison group (22\%). Furthermore, a subpopulation of impaired children with CM or M/S were particularly devastated with language and attention deficits too severe to allow testing.

In this observational study, preexisting neurologic abnormalities that predisposed to $\mathrm{M} / \mathrm{S}$ cannot be excluded. Children with underlying brain abnormalities would be more likely to present with complicated seizures in the setting of malaria and hyperthermia. Among the CM group, the possibility of subtle status epilepticus related to premorbid "bad brain" is difficult to eliminate. The only published work systematically studying EEG in CM reported 23\% of cases exhibiting sub-clinical electrographic seizures. ${ }^{4}$ Regardless of these limitations, this work should increase awareness of the potential for childhood malaria to produce chronic neurologic disorders.

Neuropsychiatric disorders comprise $>10 \%$ of the global burden of disease and the developing world is disproportionately affected. ${ }^{5}$ Severe malaria may be contributing to this burden. Could interventions aimed at neuroprotection reduce the incidence of neurologic sequelae in this population? Since severe malaria survivors almost certainly have received medical care, an intervention to diminish the damage could be broadly applied. Moreover, survivors with cognitive deficits would benefit from educational and rehabilitation services. Further work including prospective, observational studies are needed to delineate the full burden of severe malaria sequelae, increase our understanding of how malaria contributes to neurologic problems, and to begin to develop interventions that may alleviate the burden of central nervous system injuries resulting from this common infection.
J Neurol Neurosurg Psychiatry 2005;76:467-468.

doi: 10.1136/jnnp.2004.050377

Authors' affiliations

G L Birbeck, Departments of Neurology \& Epidemiology, Michigan State University, MI, USA

T E Taylor, College of Osteopathic Medicine's Department of Internal Medicine, Michigan

State University

Correspondence to: Gretchen L Birbeck, Assistant Professor, Departments of Neurology \& Epidemiology, 138 Service Road, A217, East Lansing, Ml 48824-1313, USA;

Gretchen.Birbeck@ht.msu.edu

Competing interests: none declared

\section{REFERENCES}

1 Snow RW, Craig M, Deichmann U, et al. Estimating mortality, morbidity and disability due to malaria among Africa's non-pregnant population.[see comment]. Bulletin of the World Health Organization 1999;77(8):624-40.

2 Carter J, Mung'ala-Odera V, Neville B, et al. Persistent neurocognitive impairments associated with severe falciparum malaria in Kenyan children. J Neurol Neurosurg Psychiatry 2004:75:476-81.

3 IOM. Neurological and Psychiatric Disorders: Meeting the Challenge in the Developing World. Washington DC: Institute of Medicine, 2001

4 Crawley J, Smith S, Muthinii P, et al. Electroencephalographic and clinical features of cerebral malaria. Arch Dis Child 2001;84:247-53.

5 Menken M, Munsat TL, Toole JF. The global burden of disease study: implications for neurology. Archives of Neurology 2000;57:418-20. 